\title{
The importance of communication between cyclists and other traffic participants and its potential in reducing traffic safety-critical events
}

\author{
Christine Chaloupka-Risser ${ }^{a}$, Elisabeth Füssl ${ }^{b}$ \\ ${ }^{a}$ FACTUM Chaloupka \& Risser OG, Hermann-Bahr-Straße 9/11, Vienna 1210, Austria \\ ${ }^{b}$ FACTUM Chaloupka \& Risser OG, Hermann-Bahr-Straße 9/11, Vienna 1210, Austria
}

\begin{abstract}
Over 7,600 pedestrians and cyclists died on European roads in 2013. The pressing question is how traffic safety for bicyclists can be increased? Communication is essential in traffic in order to inform other road users and to coordinate their behaviour. The current paper focuses on developing a methodology to analyse cyclists' communication resp. interaction with car drivers and other traffic participants in order to obtain a better understanding of critical events. The relation of communication and infrastructure will be discussed. The general hypothesis is that infrastructure has a marked influence on the communication between cyclists and other road users. It emphasizes the necessity and chances for communication: e. g. a good intersection design enhances the visibility of cyclists.
\end{abstract}

KEYWORDS: Naturalistic cycling; safety critical events; communication; road infrastructure; cyclists

\section{INTRODUCTION}

The main aim of the paper is to discuss the theoretical framework regarding definition of safety-critical events and the relevance of communication between different traffic participants of the BIKEALYZE project. The Austrian project BIKEALYZE (founded by the Austrian Ministry of Transportation) which started 2015, includes a naturalistic cycling study in the cities of Vienna and Salzburg. Data will be collected by (1) mobile eye tracking, (2) GPS-based motion data acquisition complemented with acceleration and steering direction data, (3) video-based data and 4) elicitation interviews. The paper will focus only on the observation and mobile eye tracking data.

\section{CYCLING SAFETY AND SAFETY POLICY}

Over 7,600 pedestrians and cyclists died on European roads in 2013. Cycling is an increasingly popular alternative transport mode, but there were far too many cycling fatalities over the past decade (European Transport Safety Council 2015). 138,400 pedestrians \& cyclists were killed in EU between 2001-2013. According to recent safety analysis data from ETSC (2015) bicyclists face a high accident risk. Vulnerable road users account for $29 \%$ of all road deaths across EU (pedestrians: 21\%, cyclists: $8 \%$ ). Not only that they face a high risk, there is a slower decrease in the rate of deaths of unprotected road users than for motor vehicle users (vehicle occupants: 53\% compared to pedestrians: $41 \%$, cyclists: $37 \%)$. According to a TRL report (Knowles et al 2008) more than a quarter of all cycling deaths in 2005-07 happened when a vehicle ran into the rear of a bike. This occurred to more than one-third in rural areas and to $40 \%$ in collisions that took place away from junctions. According to the same report it seemed that one cause for cyclists' accidents was that car drivers as well as cyclists "failed to look properly" - whatever the causes have been. The main question therefore is: How can traffic safety for bicyclists be enhanced?

According to the findings of past studies, cycle safety policies need to pay close attention to speed reduction of motorists in general (Knowles et al 2008) and as a consequence to the harmonization of speed of different road users. To reach these preconditions for safer cycling (mainly at intersections) the key design principles should be: support visibility and predictability especially of the so called unprotected road users and facilitate the communication between road users (Chaloupka et al. 2011). In order to support the implementation of these relevant design prin- 
ciples a methodology for collecting evidence based data about critical events and its relation towards infrastructure was developed within the BIKEALYZE project. Thus, a naturalistic cycling study including different methods were used in order to make traffic safety critical events more transparent and understandable. The development of this methodology will be described in the following.

\section{NATURALISTIC CYCLING STUDIES IN AUSTRIA - THE PROJECT BIKEALYZE}

Naturalistic cycling studies have gained considerable attention internationally as a useful scientific method to study the interaction of cyclists in their environment in a naturalistic manner. So far naturalistic cycling studies were not established in Austria. Within the BIKEALYZE project naturalistic cycling data from 24 test participants during two experiment runs in Salzburg and Vienna will be collected. Special attention has been paid to safety-critical events (e.g. collision avoidance, way-giving violations, abrupt braking, abrupt turnout) and communication problems. In the first part of data collection each participant will collect motion data and video data for the period of one week. In the second part 12 participants in Salzburg will collect mobile eye tracking data. Safety-critical events will then be extracted from the recorded data. These events will be categorized and mapped onto a digital map representing the existing cycling infrastructure in Salzburg and in Vienna.

\section{METHODOLOGY}

\subsection{Concept of critical events}

As crash data show (see chapter 2), cycling safety is a pressing issue. In order to prevent cyclists from crash it is necessary to focus on problems in traffic that occur before crash happens (Hydén 1987). These critical events in traffic are embedded in communication processes (resp. behaviour), but there is no standardized methodology how to identify critical events. Within the BIKEALYZE project such a methodology will be developed. In the following the theoretical background of the developed methodology is presented: 1) psychophysical conditions of critical events, 2) concept of comfort and safety, 3) concept of communication, 4) causing factors for critical events and 5) a systematic for identifying accident causation.

\section{Safety-critical events in traffic - in general}

Safety-critical events arise in the context of interaction between persons and/or environment. Besides the objective safety risk, the subjective feeling of (un)safety (fear of accidents) related to man (the cyclist) has to be considered alongside usability issues (related to infrastructure) including comfort.

Crash data only contain "objective" data but never "subjective safety" elements. The latter often are responsible for misinterpretation of special situations or infrastructure conditions. Considering the holistic assessment of the traffic situation (i.e. subjective and objective safety), it is necessary to distinguish between two levels

- Perception of persons: regarding safety, accessibility, comfort and

- Objective situation: data about accidents/ safety, accessibility etc.

Both levels do not necessarily match and may differ also in different drivers/riders because of psychophysical condition, experience etc. (e.g. everyday trips, children, parents with children, elderly etc.). Therefore: both levels have to be considered and examined separately with different qualitative \& quantitative methods.

\subsection{Comfort and safety}

"Comfort" can be defined as a positive emotional reaction to external conditions. Factors influencing comfort implicate the satisfaction on at least four different levels (Øvstedal et al. 2002):

- Physiological needs: enough space and light

- Needs of safety: sufficient subjective safety, amply time to cross an intersection (before cars turn in etc.)

- Cognitive needs: sufficient support for orientation and communication

- Aesthetical needs: appealing design, no dirt on the pavement etc.

Infrastructural conditions could cause discomfort and evoke safety-critical events because of the restrictions in communication. For example, a cyclist has to leave the dedicated cycling infrastructure and has to negotiate the way alongside of cars (see Fig. 1), having to handle the speed difference and accept the driver's disregard of rules. Or cyclists have to compensate for shortcomings in infrastructure, such as disturbed intervisibility. 

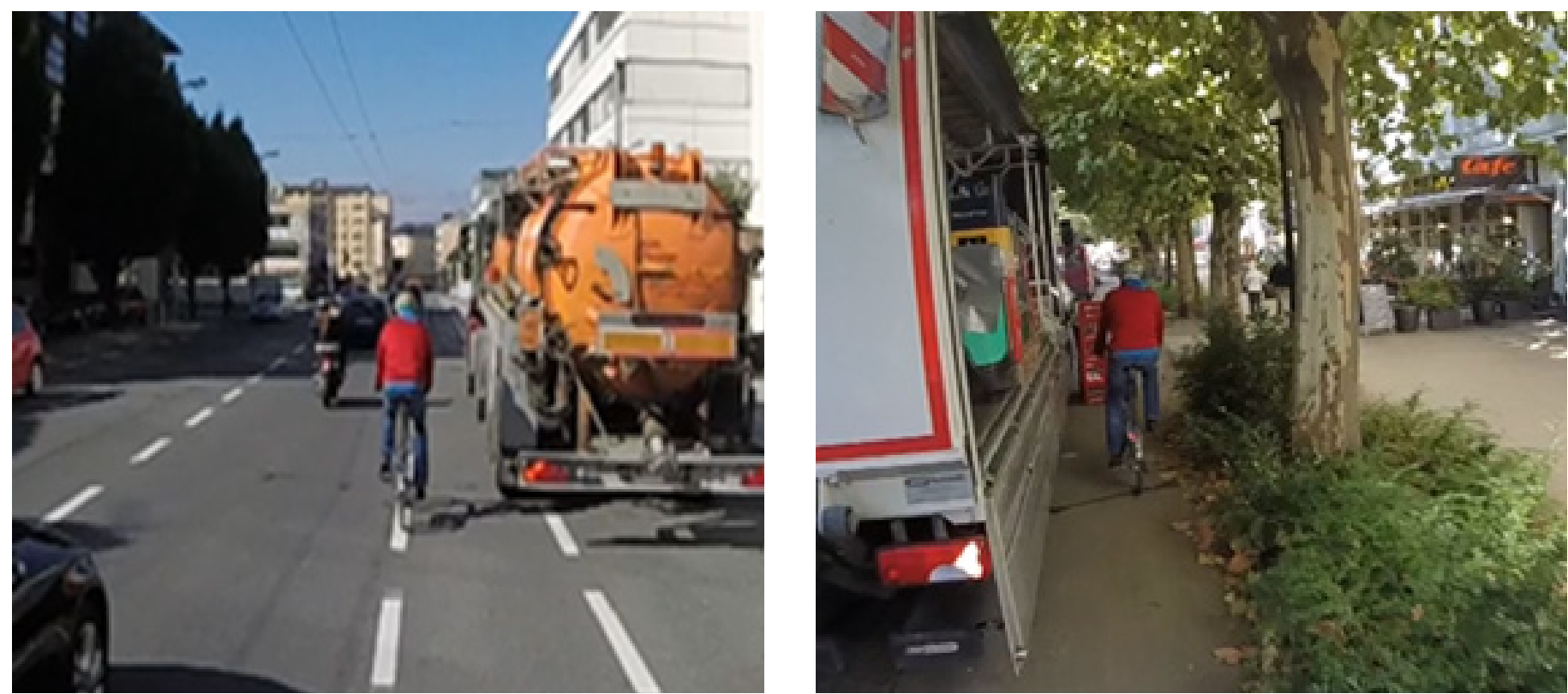

Figure 1: Cyclist has to be highly attentive and communicate because of barriers on the cycle track (C) Salzburg Research GmbH)

\subsection{Communication in traffic}

Communication is necessary in traffic in order to inform other road users about one's intentions and needs, to coordinate mutual actions and to give feedback if a situation/interaction tends to be complicated, uncomfortable or unsafe. Besides formal rules, informal rules play an important role in road traffic as they guide road users' actions. Communication is not always helpful or friendly. Some examples of problematic communication especially between car drivers and cyclists are following ${ }^{\mathbf{1}}$ :

- other road users (e.g. car drivers honking the horn to chase off cyclists of their way; the overtake with narrow distance which can be interpreted as a sign for disrespect),

- infrastructure (e.g. cycle infrastructure (and view) is obstructed by (parked) cars)

- other road users and infrastructure (e.g. cycling on the pavement because of missing cycling infrastructure; ruthlessness of car drivers)

For some cyclists such experiences of problematic communication might negatively affect their feeling of comfort and safety (Sanders 2015).

1 Source: Austrian Science Fund (Project n P 26192 - G22) "Interaction between cyclists and car drivers"; in depth interview data. September 2014 - April 2015

\subsection{Main factors causing safety-critical events Factor ENVIRONMENT}

Black spots show where the interplay of human and infrastructure does not function in an optimal way. However, the environmental conditions are rarely the only reason for crashes or traffic conflicts. On the contrary, safety relevant events occur often within the process of interaction between different road users. Especially at intersections where special attention is required when negotiating a limited space between all traffic participants.

\section{HUMAN factor}

According to many international studies human failure is responsible for accidents in traffic in approx. $90 \%$ of cases (NHTSA 2008; Singh 2015). For the development of the BIKEALYZE methodology a "tool" was chosen which allows to differentiate between different steps of the human information processing performance. As communication is a process of information exchange, the "Accident Causation Analysis System" (ACAS) - tool was considered as a valuable hint at which levels within the information processing operation problems may occur which lead to critical events.

The following part gives a short introduction to the ACAS (Otte et al. 2009), which was choosen to serve as a model for the investigation and explanation of critical events for cyclists. As ACAS shows, within the communication process various tasks have to be completed, failure might lead to conflicts or crash. 


\subsection{ACAS Categories}

The ACAS (Otte et al. 2009) differentiates between phases of information processing where errors may arise, as well as human misbehaviour, conflicts or crash.

ACAS is a tool for collecting human error causation data, which describes the human information processing factors in a chronological sequence. According to findings of accident analysis studies, which used this tool to explore relevant issues for countermeasures, it appears that the ability to recognize information subjectively proved to be the main source of errors in traffic (Otte et al. 2009). This corresponds with findings of the NHTSA (Singh, 2015) which show that the recognition error, consisting of driver's inattention, internal and external distractions, and inadequate surveillance, was the most frequently recorded critical reason $(41 \% \pm 2.2 \%)$.

\section{OPERATIONALISATION}

The following section will give an overview of the operationalisation of 'critical events' related to information admission and will illustrate how interaction data is registered by the different methods in the BIKEALYZE project. As 'information admission' is the most problematic part within the process of information processing (see Figure 2), this category has been used to illustrate the development of the methodology for identifying critical events.

\subsection{Operationalization of the category "information admission" according to ACAS}

The main question related to this category is, "could all relevant information be recognized objectively and subjectively?". The baseline hypothesis ( $\mathrm{H} 0)$ therefore is: Necessary information is available for the bicyclist at least objectively and it is possible to be recognized by the cyclist objectively (according to Otte et al 2009).

The following overview will give information about the assumed possibilities to operationalize some aspects of the category "information admission" by different technological tools as described above.

\subsection{Method: Video observation}

When it comes to bicycle studies in natural environment, video recordings are quite popular and therefore frequently used because they are cost-efficient and an easy way of using a method which is able to provide highly precise information of the driving situation. In the current study helmet cameras (GO PRO action cams) were used for data collection (see Johnson et al. 2010). The cameras, pointing in the driving direction, were fitted on the helmets of the test persons. Thus the cyclists' line of sight and the surrounding situational context like traffic density,

\section{Information access \\ Was the driver able to detect objectively all relevant information? \\ Information admission Was the driver able to identify subjectively every information which is relevant for the traffic situation? \\ Information evaluation Having every available information at hand, was the driver able to correctly assess the situation? \\ Planning \\ Was the choice the driver made appropriate for the given situation? \\ Operation \\ Was the driver able to translate the chosen action into correct operation?}

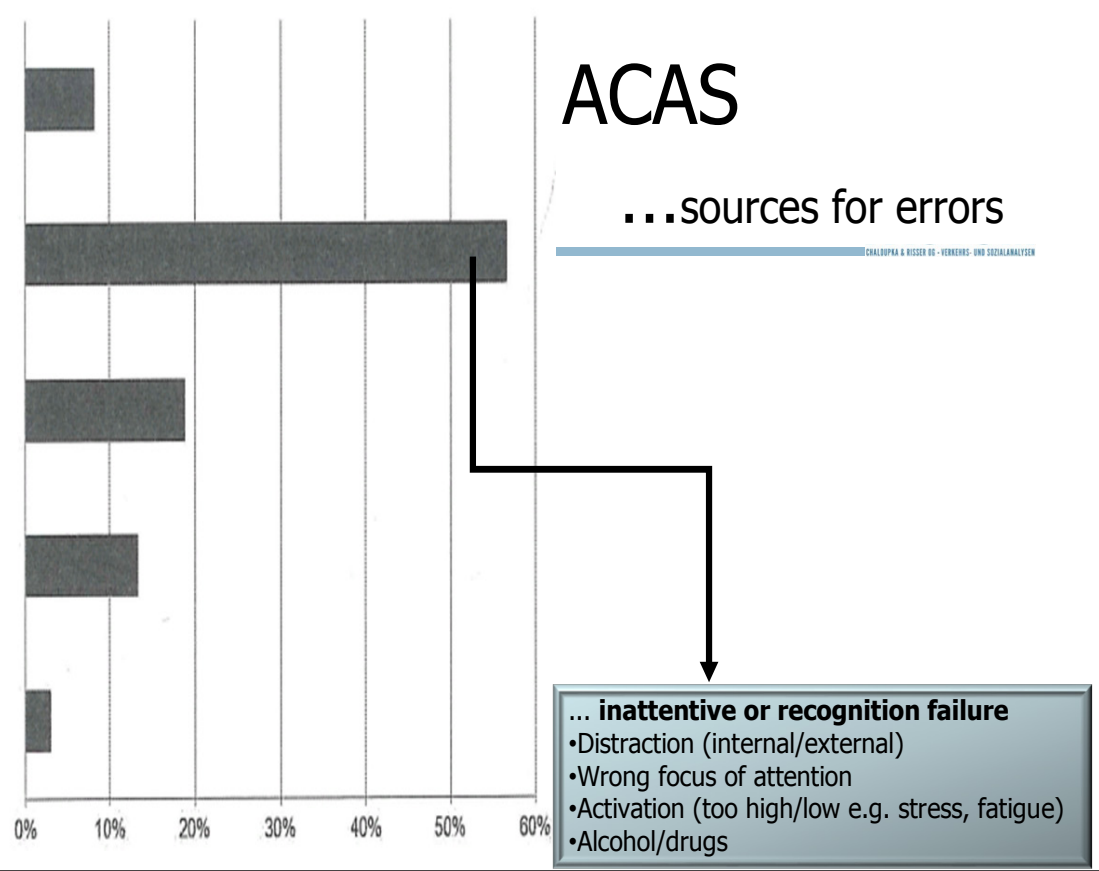


Table 1: Overview of possible methods for operationalization of behaviour related to lack of information admission in the road infrastructure and to elements obstructing information admission

\begin{tabular}{ll}
\hline Elements in road infrastructure and behaviour that affect information admission & Appropriate methods \\
\hline Barriers for orientation: (traffic) signs, trees etc. & Video, interrogation, eye tracking \\
Lacking orientation points & Video, interrogation, eye tracking \\
Deceleration before intersection or encounter & Video \\
Distraction because of special traffic sounds, mobile phone, shop window etc. & Video, eye tracking, interrogation \\
Distraction because of maintenance of bicycle (e.g. mud guard broken) & Video, eye tracking, interrogation \\
Incorrect focus of attention (fixation on objects/events that are not relevant & Eye tracking, Video \\
for the situation) & \\
Uncertain lane keeping & Video, eye tracking \\
\hline
\end{tabular}

physical barriers or weather conditions could be captured. Former studies discussed the advantages and disadvantages of certain positions for fitting of the camera (e.g. camera mounted on handlebar see Gustafsson \& Archer 2013, Dozza et al. 2012, or usage of helmet camera see Johnson et al. 2010). The decisive advantage of cameras mounted on the helmet was, that the conditions for cycling were still naturalistic as the test riders did not need to equip themselves with additional accessories, e.g. chest belt. An additional advantage of helmet cameras is that one is able to register head moves (turning of head) which are an important way for cyclists to either communicate with other road users and/or to collect information from the surrounding.

\subsection{Method: Mobile Eye-tracking}

Mobile Eye Tracking (MET) data is used in order to analyse gazes of persons in specific environment. In naturalistic cycling studies these data provide information about the areas bikers fixate, the direction and duration of their gazes. So called areas of interest (AOI) can be defined in order to investigate if persons notice specific elements in their environment. Recently eye tracking has been used in geoinformatic studies (e.g. Brychtová A. \& Coltekin A. 2014, Popelka 2016) but scarcely in naturalistic cycling studies. In the BIKEALYZE project the eye tracking data is collected with Tobii Pro Glasses 2. The glasses record gaze data which include fixations as well as saccades. Additional various metrics about the gaze data, e.g. interval duration and area of interest (AOI) and specific metrics can be deduced from the data when the analysis software Tobii Pro Glass Analyser has been used. The use of MET for naturalistic cycling studies is an uncharted field in traf- fic science; except for a view studies, see Vansteenkiste et al. 2013, there is hardly any reference available.

\subsection{Elicitation interviews}

During video elicitation interviews, the researchers will interview the participants about their subjective feeling of safety while riding on this specific route and their feeling of comfort by using a video recording of that cycling trip (including all interactions and critical events) as an elicitation tool. The participants will inform the researchers if they had recognized any information admission problems on the trip and what they had perceived as critical events.

\subsection{Relation between category "information admission" and "communication"}

Conclusions out of specific detected behaviour according to Table 1 therefore could be: an uncertain lane keeping might indicate lack of information access or processing (error in communication or coordination with other traffic participants). This is relevant as information (e.g. for orientation) has to be provided in due time so that it can be processed in a short time e.g. if the cycle lane ends (see Fig. 3). So if there is lack of information, the time to coordinate one's behaviour with other road users will be limited or there will be none at all. In the worst case this may result in a crash.

The gaze plot in Figure 3 gives an impression about data obtained from the eye tracker (Tobii glasses) showing the time span of 1,92 sec of approach of a cyclist to an intersection. The arrow in the middle of the picture shows the way the cyclist has to take and shows his searching activities within this very short time span. 


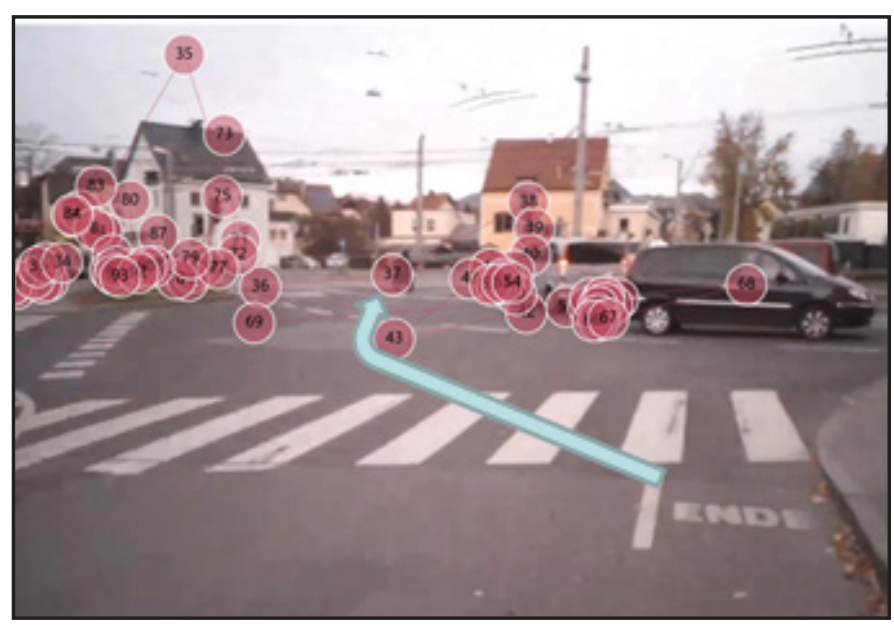

Figure 3: Eye tracking Gaze Plot (interval length of all gazes $=1,92$ sec.): Was the relevant information perceived? (C) FACTUM \& Salzburg Research)

\section{CONCLUSIONS}

This study has presented the development of a methodology for identifying critical events as an important indicator for improving infrastructure and prevent crash. The combined use of different data sources enables an innovative approach when interactions in traffic are to be analysed. Interaction and behaviour data, infrastructural conditions and gaze behaviour data can be interrelated and can finally provide an improved understanding of their reciprocal influence on each other. It should underline the importance of considering communication in naturalistic cycling studies and provide a more informative and complete picture of events which are relevant for comfort and safety. Thus, we are able to consider the complexity of the traffic system.

The obtained results can be adapted and used for drivers' training, but also for the training of cyclists and can subsequently target the improvement of communication between different road users. In addition, recommendations for the adaptation and/ or improvement of cycling infrastructure or other inputs for road safety awareness campaigns can be also derived from the results.

A challenging issue with regard to analysing communication and critical events based on different methods is the integration of the resulting data (video data, eye tracking data and interview data) into a joint category system. This is a time consuming task, as concepts and theoretical approaches have to be linked considering their epistemological foundation. Especially the use of eye tracking data in the field of traffic is a rather new issue and much further research is necessary to clarify what the instrument eye tracking glass is exactly able to measure.

\section{REFERENCES}

Brychtová, A., Coltekin, A. (2014): An Empirical User Study for Measuring the Influence of Colour Distance and Font Size in Map Reading Using Eye Tracking. The Cartographic Journal Maney Publishing, 14s.

Chaloupka-Risser, C., Risser, R., Zuzan, W. (2011) Verkehrspsychologie: Grundlagen und Anwendungen. Wien: Facultas.

Gustafsson, L., \& Archer, J. (2013). A naturalistic study of commuter cyclists in the greater Stockholm area. Accident; Analysis and Prevention, 58, 286-98. Retrieved from http://www.sciencedirect.com/science/article/pii/S0001457512002266.

Hydén, Ch. (1987) The development of a method for traffic safety evaluation: The Swedish Traffic Conflicts Technique. Bulletin 70. Department of Traffic Engineering, Lund University.

Johnson, M., Charlton, J., Oxley, J., \& Newstead, S. (2010). Naturalistic cycling study: identifying risk factors for on-road commuter cyclists. In Annals of Advances in Automotive Medicine/Annual Scientific Conference, 54, 275-83. Association for the Advancement of Automotive Medicine. Retrieved from http://www.pubmedcentral.nih.gov/articlerender.fcgi?artid=3 242541\&tool=pmcentrez\&rendertype $=$ abstract.

Knowles, J., Adams, R., Cuerden, R., Savill, T., Reid, S. and Tight, M. (2008). Collisions Involving Cyclists on Britain's Roads: Establishing the Causes, TRL (PPR 445).

NHTSA (2008). National Motor Vehicle Crash Causation Survey, Report to congress No. DOT HS 811 059. Washington, DC: National Highway Traffic Safety Administration

Øvstedal, L., Ryeng,E.O. (2002): Understanding Pedestrian Comfort in European Cities: How to improve walking conditions? Paper presented at the European Transport Conference 2002, September 9-11 2002, Homerton College, Cambridge, UK.

Otte, D., Pund, B., Jänsch, M. (2009): Unfallursachen-Analyse ACAS für Erhebungen am Unfallort. In: Zeitschrift für Verkehrssicherheit, 122-128.

Popelka, St. (2016). Eye-tracking with dynamic stimuli. Dep. of Geoinformatics, University of Olomouc.

Sanders, R. L. (2015). Perceived traffic risk for cyclists: The impact of near miss and collision experiences. Accident Analysis \& Prevention 75, 26-34. doi: 10.1016/j.aap.2014.11.004.

Singh, S. (2015). Critical reasons for crashes investigated in the National Motor Vehicle Crash Causation Survey. Traffic Safety Facts Crash•Stats. Report No. DOT HS 812 115. Washington, DC: National Highway Traffic Safety Administration.

Vansteenkiste, P., Cardon, G., \& Lenoir, M. (2013). Dealing with head-mounted eye-tracking data. In Proceedings of the 2013 Conference on Eye Tracking South Africa ETSA, 13, 55-57. New York, New York, USA: ACM Press. doi:10.1145/2509315.2509325. 


\section{Web sources}

European Transport Safety Council 2015: Europe must boost safety for cyclists and walkers

http://etsc.eu/europe-must-boost-safety-for-cyclists-and-walkers/ (27. 6. 2016)

European Transport Safety Council 2015: PIN Flash 29 Making walking and cycling on Europe's roads safer, p. 17

http://etsc.eu/wp-content/uploads/etsc_pin_flash_29_walking_cycling_safer.pdf $(27.6 .2016)$ 